\title{
Convergence of general algorithm for I-generalized asymptotically nonexpansive nonself-mappings in uniformly convex hyperbolic spaces
}

Liping Yang*

\section{"Correspondence:} yanglping2003@126.com School of Applied Mathematics, Guangdong University of Technology, Guangzhou, 510520, China

\begin{abstract}
In this paper, a new iterative scheme for a finite family of $l_{i}$-generalized asymptotically nonexpansive nonself-mappings $\left\{T_{i}\right\}_{i=1}^{r}$ is constructed in a uniformly convex hyperbolic space. We establish strong convergence theorems of this iterative scheme to a common fixed point of $\left\{T_{i}\right\}_{i=1}^{r}$ and $\left\{l_{i}\right\}_{i=1}^{r}$ under certain conditions. Our results of this paper extend some results in the literature.
\end{abstract}

MSC: $47 \mathrm{H} 09 ; 47 \mathrm{H} 10$

Keywords: I-generalized asymptotically nonexpansive nonself-mappings; common fixed point; iteration scheme; hyperbolic spaces; $\triangle$-convergence

\section{Introduction}

Let $T: K \rightarrow K, I: K \rightarrow K$ be two mappings of nonempty subset $K$ of a real normed linear space $X . T$ is said to be $I$-asymptotically nonexpansive $[1,2]$ if there exists a sequence $\left\{v_{n}^{\prime}\right\} \subset[0, \infty)$ with $\lim _{n \rightarrow \infty} v_{n}^{\prime}=0$ such that

$$
\left\|T^{n} x-T^{n} y\right\| \leq\left(1+v_{n}^{\prime}\right)\left\|I^{n} x-I^{n} y\right\|
$$

for all $x, y \in K$ and $n \geq 1$.

A subset $K$ of $X$ is said to be a retract of $X$ if there exists a continuous map $P: X \rightarrow K$ such that $P x=x, \forall x \in K$. A mapping $P: X \rightarrow K$ is said to be a retraction if $P^{2}=P$. It follows that if a map $P$ is a retraction, then $P y=y$ for all $y$ in the range of $P$.

For nonself-nonexpansive mappings, some authors (see $[3,4]$ and the references therein) have studied the strong and weak convergence theorems in Hilbert spaces or uniformly convex Banach spaces. However, iterative algorithms for approximating fixed points of nonself-asymptotically nonexpansive mappings have not been paid too much attention. The concept of nonself-asymptotically nonexpansive mappings was introduced by Chidume et al. [5] in 2003 as a generalization of asymptotically nonexpansive selfmappings.

(c) 2015 Yang. This article is distributed under the terms of the Creative Commons Attribution 4.0 International License (http://creativecommons.org/licenses/by/4.0/), which permits unrestricted use, distribution, and reproduction in any medium, provided you give appropriate credit to the original author(s) and the source, provide a link to the Creative Commons license, and indicate if changes were made. 
The concept of asymptotically nonexpansive nonself-mappings in the intermediate sense was introduced by Chidume et al. [6] as an important generalization of asymptotically nonexpansive self-mappings in the intermediate sense.

Definition 1.1 ([6]) Let $K$ be a nonempty subset of a Banach space $X$. Let $P: X \rightarrow K$ be a nonexpansive retraction of $X$ onto $K$. A nonself-mapping $T: K \rightarrow X$ is called asymptotically nonexpansive in the intermediate sense if $\mathrm{T}$ is continuous and the following inequality holds:

$$
\limsup _{n \rightarrow \infty} \sup _{x, y \in K}\left(\left\|T(P T)^{n-1} x-T(P T)^{n-1} y\right\|-\|x-y\|\right) \leq 0 .
$$

By studying the following iterative sequence:

$$
x_{n+1}=P\left(\left(1-\alpha_{n}\right) x_{n}+\alpha_{n} T(P T)^{n-1} x_{n}\right), \quad \forall x_{1} \in K, n \geq 1,
$$

Chidume et al. [5] established demi-closed principle, strong and weak convergence theorems for nonself asymptotically nonexpansive mapping in a uniformly convex Banach space. Recently concerning the convergence problem of an explicit iterative process to a common fixed point for some nonself-asymptotically nonexpansive mappings in uniformly convex Banach spaces have been considered by several authors (see, for example, Wang [7], Yang [8], Thainwan [9], and the references therein).

Inspired and motivated by those facts, we will construct a new type of iterative sequence involving $I$-generalized asymptotically nonexpansive nonself-mapping in a nonempty closed convex subset of complete uniformly convex hyperbolic spaces and study the strong convergence for such mappings. Our theorems improve and generalize important related results of the previously known ones announced by Chidume et al. [6], Wang [7].

\section{Preliminaries}

Definition 2.1 Let $(X, d)$ be a metric space and $T: K \rightarrow X, I: K \rightarrow X$ be two mappings of nonempty subset $K$ of $X$. A nonself-mapping $T: K \rightarrow X$ is said to be $I$-generalized asymptotically nonexpansive mapping if there exist sequences $\left\{v_{n}\right\} \subset[0, \infty)$ and $\left\{s_{n}\right\} \subset$ $[0, \infty)$ with $\lim _{n \rightarrow \infty} v_{n}=0=\lim _{n \rightarrow \infty} s_{n}$ such that

$$
d\left(T(P T)^{n-1} x, T(P T)^{n-1} y\right) \leq\left(1+v_{n}\right) d\left(I(P I)^{n-1} x, I(P I)^{n-1} y\right)+s_{n}
$$

for all $x, y \in K$, where $P$ is a nonexpansive retraction of $X$ onto $K$.

If $I$ is the identity mapping, then (2.1) reduces to

$$
d\left(T(P T)^{n-1} x, T(P T)^{n-1} y\right) \leq\left(1+v_{n}\right) d(x, y)+s_{n}
$$

then $T$ is said to be generalized asymptotically nonexpansive nonself-mapping.

$T: K \rightarrow X$ is said to be uniformly $L$-Lipschitzian if there exists a constant $L>0$ such that

$$
d\left(T(P T)^{n-1} x, T(P T)^{n-1} y\right) \leq L d(x, y), \quad \forall n \geq 1, x, y \in K .
$$

Remark 2.2 If $s_{n}=0$ for all $n \geq 1$, then (2.1) reduces to the nonself-asymptotically nonexpansive mapping. If $v_{n}=0$ for all $n \geq 1$ and $I$ the identity mapping, then $I$-generalized 
asymptotically nonexpansive nonself-mappings coincide with asymptotically nonexpansive nonself-mappings in the intermediate sense.

A hyperbolic space [10] is a metric space $(X, d)$ together with a map $W: X^{2} \times[0,1] \rightarrow X$ satisfying:

(i) $d(u, W(x, y, \lambda)) \leq \lambda d(u, x)+(1-\lambda) d(u, y)$,

(ii) $d(W(x, y, \lambda), W(x, y, \mu))=|\lambda-\mu| d(x, y)$,

(iii) $W(x, y, \lambda)=W(y, x,(1-\lambda))$,

(iv) $d(W(x, z, \lambda), W(y, w, \lambda)) \leq(1-\lambda) d(x, y)+\lambda d(z, w)$,

for all $x, y, z, w \in X$ and $\lambda, \mu \in[0,1]$. We denote the above defined hyperbolic space by $(X, d, W)$; if it satisfies only (i), then it is said to be the convex metric space introduced by Takahashi [11]. A nonempty subset $K$ of a hyperbolic space $X$ is convex if $W(x, y, \lambda) \in K$ for all $x, y \in X$ and $\lambda \in[0,1]$.

Kohlenbach [10] pointed out that all normed spaces and their subsets are hyperbolic spaces as well as convex metric spaces. The class of hyperbolic spaces is properly contained in the class of convex metric spaces.

A hyperbolic space $(X, d, W)$ is said to be uniformly convex [12] if for any $x, y, u \in X$, $r>0$ and $\epsilon \in(0,2]$, there exists a $\delta \in(0,1]$ such that

$$
\left.\begin{array}{l}
d(x, u) \leq r \\
d(y, u) \leq r \\
d(x, y) \geq \epsilon r
\end{array}\right\} \quad \Rightarrow \quad d\left(W\left(x, y, \frac{1}{2}\right), u\right) \leq(1-\delta) r .
$$

A map $\eta:(0, \infty) \times(0,2] \rightarrow(0,1]$ which provides such a $\delta=\eta(r, \epsilon)$, for given $r>0$ and $\epsilon \in(0,2]$, is called modulus of uniform convexity of $X$. We call $\eta$ monotone if it decreases with $r$ (for a fixed $\epsilon$ ). We call $\eta$ monotone if it decreases with $r$ (for a fixed $\epsilon$ ).

The concept of $\triangle$-convergence in a metric space was introduced by Lim [13] and its analog in $C A T(0)$ spaces has been investigated by Dhompongsa and Panyanak [14]. In this article, we continue the investigation of $\triangle$-convergence in the general setup of hyperbolic spaces.

Let $\left\{x_{n}\right\}$ be any bounded sequence in a metric space $X$. For $x \in X$, define a continuous function $\widetilde{r}\left(\cdot,\left\{x_{n}\right\}\right): X \rightarrow[0, \infty)$ by

$$
\widetilde{r}\left(x,\left\{x_{n}\right\}\right)=\limsup _{n \rightarrow \infty} d\left(x, x_{n}\right)
$$

The asymptotic radius $\rho=\widetilde{r}\left(x_{n}\right)$ of $\left\{x_{n}\right\}$ with respect to a subset $K \subset X$ is given by

$$
\rho=\inf \left\{\widetilde{r}\left(x,\left\{x_{n}\right\}\right): x \in K\right\} .
$$

The asymptotic center of a bounded sequence $\left\{x_{n}\right\}$ with respect to a subset $K \subset X$ is defined as follows:

$$
A_{K}\left(x_{n}\right)=\left\{x \in X: \widetilde{r}\left(x,\left\{x_{n}\right\}\right) \leq \widetilde{r}\left(y,\left\{x_{n}\right\}\right) \text { for any } y \in K\right\} .
$$

If the asymptotic center is taken with respect to $X$, then it is simply denoted by $A\left(x_{n}\right)$. In general, $A\left(x_{n}\right)$ may be empty or may even contain infinitely many points. It is well known that a complete uniformly convex hyperbolic space with monotone modulus of uniform 
convexity enjoys the property that bounded sequences have unique asymptotic center with respect to closed convex subsets [15].

A sequence $\left\{x_{n}\right\}$ is said to $\Delta$-convergence $x \in X$ if $x$ is the unique asymptotic center for every subsequence $\left\{x_{n_{i}}\right\}$ of $\left\{x_{n}\right\}$. In this case, we call $x$ as $\Delta$-limit of $\left\{x_{n}\right\}$ and write $\triangle-\lim _{n} x_{n}=x$.

Lemma 2.3 (see [15]) Let $(X, d, W)$ be a uniformly convex hyperbolic space with monotone modulus of uniform convexity and $K$ a nonempty closed convex subset of $X$. Then every bounded sequences $\left\{x_{n}\right\}$ in $X$ has a unique asymptotic center with respect to $K$.

Lemma 2.4 (see [15]) Let $(X, d, W)$ be a uniformly convex hyperbolic space with monotone modulus of uniform convexity $\eta$ and $x \in X$. Let $\left\{\lambda_{n}\right\} \in[b, c]$ for some $b, c \in(0,1)$. If $\left\{u_{n}\right\}$ and $\left\{v_{n}\right\}$ are sequences in $X$ such that $\lim _{\sup _{n \rightarrow \infty}} d\left(u_{n}, x\right) \leq r, \limsup _{n \rightarrow \infty} d\left(v_{n}, x\right) \leq r$ and $\lim _{n \rightarrow \infty} d\left(W\left(u_{n}, v_{n}, \lambda_{n}\right), x\right)=r$ for some $r \geq 0$, then $\lim _{n \rightarrow \infty} d\left(u_{n}, v_{n}\right)=0$.

Lemma 2.5 (see [15]) Let $K$ a nonempty closed convex subset of a uniformly convex hyperbolic space $(X, d, W)$ and $\left\{u_{n}\right\}$ be a bounded sequence in $K$ such that $A\left(\left\{u_{n}\right\}\right)=\{u\}$. If $\left\{v_{m}\right\}$ is any other sequence in $K$ such that $\lim _{m \rightarrow \infty} r\left(v_{m},\left\{u_{n}\right\}\right)=r\left(u,\left\{u_{n}\right\}\right)$, then $\lim _{m \rightarrow \infty} v_{m}=u$.

Lemma 2.6 (see [16], Lemma 1) Let $\left\{a_{n}\right\},\left\{b_{n}\right\},\left\{\delta_{n}\right\}$ be sequences of nonnegative real numbers satisfying the inequality

$$
a_{n+1} \leq\left(1+\delta_{n}\right) a_{n}+b_{n}, \quad n \geq 1
$$

If $\sum_{n=1}^{\infty} b_{n}<\infty$ and $\sum_{n=1}^{\infty} \delta_{n}<\infty$, then

(i) $\lim _{n \rightarrow \infty} a_{n}$ exists;

(ii) in particular, if $\left\{a_{n}\right\}$ has a subsequence $\left\{a_{n_{k}}\right\}$ converging to 0 , then $\lim _{n \rightarrow \infty} a_{n}=0$.

In the following, we denote $I_{0}=\{1,2, \ldots, r\}$.

A family $\left\{T_{i}: i \in I_{0}\right\}$ be $I_{i}$-generalized asymptotically nonexpansive nonself-mappings and $\left\{I_{i}: i \in I_{0}\right\}$ be generalized asymptotically nonexpansive nonself-mappings with $\mathcal{F}=$ $\bigcap_{i=1}^{r} F\left(T_{i}\right) \cap F\left(I_{i}\right) \neq \emptyset$, are said to satisfy condition (B) with respect to the sequence $\left\{u_{n}\right\}$ if there is a nondecreasing function $f:[0, \infty) \rightarrow[0, \infty)$ with $f(0)=0, f(s)>0$ for all $s>0$ such that

$$
\max _{i \in I_{0}}\left\{d\left(u_{n}, T_{i} u_{n}\right)\right\} \vee \max _{i \in I_{0}}\left\{d\left(u_{n}, I_{i} u_{n}\right)\right\} \geq f\left(d\left(u_{n}, \mathcal{F}\right)\right) .
$$

\section{Main results}

Lemma 3.1 Let $(X, d, W)$ be a convex metric space and $K$ be a nonempty closed convex subset of $X$. Let $T_{i}: K \rightarrow X\left(i \in I_{0}\right)$ be $I_{i}$-generalized asymptotically nonexpansive nonself-mappings with sequences $\left\{v_{i n}\right\},\left\{s_{i n}\right\} \subset[0, \infty)$ and $I_{i}: K \rightarrow X\left(i \in I_{0}\right)$ be generalized asymptotically nonexpansive nonself-mappings with $\left\{u_{i n}\right\},\left\{s_{i n}^{\prime}\right\} \subset[0, \infty)$, and $\mathcal{F}=$ $\bigcap_{i=1}^{r} F\left(T_{i}\right) \cap F\left(I_{i}\right) \neq \emptyset$. Suppose that for any given $x_{1} \in K$, the sequence $\left\{x_{n}\right\}$ is generated by

$$
\left\{\begin{array}{l}
z_{n}=P W\left(I_{i}\left(P I_{i}\right)^{n-1} x_{n}, x_{n}, a_{n}\right), \\
y_{n}=P W\left(T_{i}\left(P T_{i}\right)^{n-1} z_{n}, P W\left(T_{i}\left(P T_{i}\right)^{n-1} x_{n}, x_{n}, \frac{c_{n}}{1-b_{n}}\right), b_{n}\right), \\
x_{n+1}=P W\left(T_{i}^{n-1} y_{n}, P W\left(T_{i}^{n-1} z_{n}, x_{n}, \frac{\beta_{n}}{1-\alpha_{n}}\right), \alpha_{n}\right),
\end{array}\right.
$$


satisfying the conditions:

(1) $\sum_{n=1}^{\infty} h_{n}<\infty$ and $\sum_{n=1}^{\infty} s_{n}<\infty$, where $h_{n}=\max \left\{u_{i n}: i \in I_{0}\right\} \vee \max \left\{v_{i n}: i \in I_{0}\right\}$ and $s_{n}=\max \left\{s_{i n}: i \in I_{0}\right\} \vee \max \left\{s_{i n}^{\prime}: i \in I_{0}\right\}$.

(2) $0 \leq a_{n}, b_{n}, c_{n}, \alpha_{n}, \beta_{n}, b_{n}+c_{n}, \alpha_{n}+\beta_{n} \leq 1, \forall n \geq 1$.

Then the sequence $\left\{x_{n}\right\}$ defined by (3.1) has limit existence property for the mappings $T_{i}$ and $I_{i}\left(i \in I_{0}\right)$.

Proof For any given $q \in \mathcal{F}$. It follows from (3.1) that

$$
\begin{aligned}
d\left(z_{n}, q\right) & =d\left(P W\left(I_{i}\left(P I_{i}\right)^{n-1} x_{n}, x_{n}, a_{n}\right), q\right) \\
& \leq d\left(W\left(I_{i}\left(P I_{i}\right)^{n-1} x_{n}, x_{n}, a_{n}\right), q\right) \\
& \leq a_{n} d\left(I_{i}\left(P I_{i}\right)^{n-1} x_{n}, q\right)+\left(1-a_{n}\right) d\left(x_{n}, q\right) \\
& \leq a_{n}\left[\left(1+h_{n}\right) d\left(x_{n}, q\right)+s_{n}\right]+\left(1-a_{n}\right) d\left(x_{n}, q\right) \\
& \leq\left(1+h_{n}\right) d\left(x_{n}, q\right)+s_{n} .
\end{aligned}
$$

It follows from (3.1), (3.2) that

$$
\begin{aligned}
d\left(y_{n}, q\right)= & d\left(P W\left(T_{i}\left(P T_{i}\right)^{n-1} z_{n}, P W\left(T_{i}\left(P T_{i}\right)^{n-1} x_{n}, x_{n}, \frac{c_{n}}{1-b_{n}}\right), b_{n}\right), q\right) \\
\leq & d\left(W\left(T_{i}\left(P T_{i}\right)^{n-1} z_{n}, P W\left(T_{i}\left(P T_{i}\right)^{n-1} x_{n}, x_{n}, \frac{c_{n}}{1-b_{n}}\right), b_{n}\right), q\right) \\
\leq & b_{n} d\left(T_{i}\left(P T_{i}\right)^{n-1} z_{n}, q\right)+\left(1-b_{n}\right) d\left(P W\left(T_{i}\left(P T_{i}\right)^{n-1} x_{n}, x_{n}, \frac{c_{n}}{1-b_{n}}\right), q\right) \\
\leq & b_{n}\left(\left(1+h_{n}\right) d\left(I_{i}\left(P I_{i}\right)^{n-1} z_{n}, q\right)+s_{n}\right) \\
& +\left(1-b_{n}\right)\left(\frac{c_{n}}{1-b_{n}} d\left(T_{i}\left(P T_{i}\right)^{n-1} x_{n}, q\right)+\left(1-\frac{c_{n}}{1-b_{n}}\right) d\left(x_{n}, q\right)\right) \\
\leq & b_{n}\left(\left(1+h_{n}\right)\left(\left(1+h_{n}\right) d\left(z_{n}, q\right)+s_{n}\right)+s_{n}\right) \\
& +c_{n}\left(\left(1+h_{n}\right) d\left(I_{i}\left(P I_{i}\right)^{n-1} x_{n}, q\right)+s_{n}\right)+\left(1-b_{n}-c_{n}\right) d\left(x_{n}, q\right) \\
\leq & b_{n}\left(1+h_{n}\right)^{3} d\left(x_{n}, q\right)+b_{n}\left(1+h_{n}\right)^{2} s_{n}+b_{n}\left(1+h_{n}\right) s_{n}+b_{n} s_{n} \\
& +c_{n}\left(1+h_{n}\right)^{2} d\left(x_{n}, q\right)+c_{n}\left(1+h_{n}\right) s_{n}+c_{n} s_{n}+\left(1-b_{n}-c_{n}\right) d\left(x_{n}, q\right) \\
\leq & \left(1+h_{n}\right)^{3} d\left(x_{n}, q\right)+\left(1+h_{n}\right)^{2} s_{n}+\left(1+h_{n}\right) s_{n}+s_{n} .
\end{aligned}
$$

It follows from (3.1), (3.2), and (3.3) that

$$
\begin{aligned}
d\left(x_{n+1}, q\right)= & d\left(P W\left(T_{i}\left(P T_{i}\right)^{n-1} y_{n}, P W\left(T_{i}\left(P T_{i}\right)^{n-1} z_{n}, x_{n}, \frac{\beta_{n}}{1-\alpha_{n}}\right), \alpha_{n}\right), q\right) \\
\leq & d\left(W\left(T_{i}\left(P T_{i}\right)^{n-1} y_{n}, P W\left(T_{i}\left(P T_{i}\right)^{n-1} z_{n}, x_{n}, \frac{\beta_{n}}{1-\alpha_{n}}\right), \alpha_{n}\right), q\right) \\
\leq & \alpha_{n} d\left(T_{i}\left(P T_{i}\right)^{n-1} y_{n}, q\right) \\
& +\left(1-\alpha_{n}\right) d\left(P W\left(T_{i}\left(P T_{i}\right)^{n-1} z_{n}, x_{n}, \frac{\beta_{n}}{1-\alpha_{n}}\right), q\right) \\
\leq & \alpha_{n}\left(\left(1+h_{n}\right) d\left(I_{i}\left(P I_{i}\right)^{n-1} y_{n}, q\right)+s_{n}\right)
\end{aligned}
$$




$$
\begin{aligned}
& +\beta_{n} d\left(T_{i}\left(P T_{i}\right)^{n-1} z_{n}, q\right)+\left(1-\alpha_{n}-\beta_{n}\right) d\left(x_{n}, q\right) \\
\leq & \alpha_{n}\left(1+h_{n}\right)\left(\left(1+h_{n}\right) d\left(y_{n}, q\right)+s_{n}\right)+\alpha_{n} s_{n} \\
& +\beta_{n}\left(\left(1+h_{n}\right) d\left(I_{i}\left(P I_{i}\right)^{n-1} z_{n}, q\right)+s_{n}\right)+\left(1-\alpha_{n}-\beta_{n}\right) d\left(x_{n}, q\right) \\
\leq & \alpha_{n}\left(1+h_{n}\right)^{2} d\left(y_{n}, q\right)+\alpha_{n}\left(1+h_{n}\right) s_{n}+\alpha_{n} s_{n} \\
& +\beta_{n}\left(1+h_{n}\right)\left(\left(1+h_{n}\right) d\left(z_{n}, q\right)+s_{n}\right)+\beta_{n} s_{n}+\left(1-\alpha_{n}-\beta_{n}\right) d\left(x_{n}, q\right) \\
\leq & \left(\alpha_{n}\left(1+h_{n}\right)^{5}+\beta_{n}\left(1+h_{n}\right)^{3}+\left(1-\alpha_{n}-\beta_{n}\right)\right) d\left(x_{n}, q\right) \\
& +\left(\left(1+h_{n}\right)^{4}+\left(1+h_{n}\right)^{3}+\left(1+h_{n}\right)^{2}+\left(1+h_{n}\right)+1\right) s_{n} \\
\leq & \left(1+h_{n}\right)^{5} d\left(x_{n}, q\right)+\sigma_{n} \\
= & \left(1+\rho_{n}\right) d\left(x_{n}, q\right)+\sigma_{n} .
\end{aligned}
$$

Since $\sum_{n=1}^{\infty} h_{n}<\infty$ and $\sum_{n=1}^{\infty} s_{n}<\infty$, we have $\sum_{n=1}^{\infty} \rho_{n}=\sum_{n=1}^{\infty} h_{n}\left(5+10 h_{n}+10 h_{n}^{2}+5 h_{n}^{3}+\right.$ $\left.h_{n}^{4}\right)<\infty$ and $\sum_{n=1}^{\infty} \sigma_{n}=\sum_{n=1}^{\infty}\left(\left(1+h_{n}\right)^{4}+\left(1+h_{n}\right)^{3}+\left(1+h_{n}\right)^{2}+\left(1+h_{n}\right)+1\right) s_{n}<\infty$. Applying Lemma 2.6 to (3.4), we observe that $\lim _{n \rightarrow \infty} d\left(x_{n}, q\right)$ exists for each $q \in \mathcal{F}$.

Lemma 3.2 Under the assumptions of Lemma 3.1, we have the following:

(i) If $0<\liminf _{n \rightarrow \infty} \alpha_{n} \leq \lim \sup _{n \rightarrow \infty}\left(\alpha_{n}+\beta_{n}\right)<1$, then $\lim _{n \rightarrow \infty} d\left(T_{i}\left(P T_{i}\right)^{n-1} y_{n}, P W\left(T_{i}\left(P T_{i}\right)^{n-1} z_{n}, x_{n}, \frac{\beta_{n}}{1-\alpha_{n}}\right)\right)=0$.

(ii) If $0<\liminf _{n \rightarrow \infty} b_{n} \leq \limsup _{n \rightarrow \infty}\left(b_{n}+c_{n}\right)<1$, then

$$
\lim _{n \rightarrow \infty} d\left(T_{i}\left(P T_{i}\right)^{n-1} z_{n}, P W\left(T_{i}\left(P T_{i}\right)^{n-1} x_{n}, x_{n}, \frac{c_{n}}{1-b_{n}}\right), b_{n}\right)=0 .
$$

(iii) If $0<\liminf _{n \rightarrow \infty} b_{n} \leq \limsup _{n \rightarrow \infty}\left(b_{n}+c_{n}\right)<1$,

$$
\begin{aligned}
& 0<\liminf _{n \rightarrow \infty} a_{n} \leq \limsup _{n \rightarrow \infty} a_{n}<1, \text { then } \\
& \lim _{n \rightarrow \infty} d\left(I_{i}\left(P I_{i}\right)^{n-1} x_{n}, x_{n}\right)=\lim _{n \rightarrow \infty} d\left(T_{i}\left(P T_{i}\right)^{n-1} x_{n}, x_{n}\right)=0 .
\end{aligned}
$$

Proof By Lemma 3.1, $\lim _{n \rightarrow \infty} d\left(x_{n}, q\right)$ exists for each $q \in \mathcal{F}$.

Let $\lim _{n \rightarrow \infty} d\left(x_{n}, q\right)=c$ for some $c \geq 0$. The case $c=0$ is trivial. Next, we discuss the case $c>0$.

(i) Assume that $0<\liminf _{n \rightarrow \infty} \alpha_{n} \leq \limsup _{n \rightarrow \infty}\left(\alpha_{n}+\beta_{n}\right)<1$, then there exist $\eta_{1}, \eta_{2} \in$ $(0,1)$ such that $0<\eta_{1} \leq \alpha_{n} \leq \alpha_{n}+\beta_{n} \leq \eta_{2}<1$ for all $n \geq 1$.

Since $T_{i}$ is $I_{i}$-generalized asymptotically nonexpansive mapping, it follows from (3.3) that we have

$$
\begin{aligned}
d\left(T_{i}\left(P T_{i}\right)^{n-1} y_{n}, q\right) \leq & \left(1+h_{n}\right) d\left(I_{i}\left(P I_{i}\right)^{n-1} y_{n}, q\right)+s_{n} \\
\leq & \left(1+h_{n}\right)\left(\left(1+h_{n}\right) d\left(y_{n}, q\right)+s_{n}\right)+s_{n} \\
\leq & \left(1+h_{n}\right)^{5} d\left(x_{n}, q\right)+\left(\left(1+h_{n}\right)^{3}+\left(1+h_{n}\right)^{2}\right. \\
& \left.+\left(1+h_{n}\right)+1\right) s_{n} .
\end{aligned}
$$

Therefore

$$
\limsup _{n \rightarrow \infty} d\left(T_{i}\left(P T_{i}\right)^{n-1} y_{n}, q\right) \leq c .
$$


Further, it follows from (3.2) that we have the inequality

$$
\begin{aligned}
d\left(P W\left(T_{i}\left(P T_{i}\right)^{n-1} z_{n}, x_{n}, \frac{\beta_{n}}{1-\alpha_{n}}\right), q\right) \leq & d\left(W\left(T_{i}\left(P T_{i}\right)^{n-1} z_{n}, x_{n}, \frac{\beta_{n}}{1-\alpha_{n}}\right), q\right) \\
\leq & \frac{\beta_{n}}{1-\alpha_{n}} d\left(T_{i}\left(P T_{i}\right)^{n-1} z_{n}, q\right) \\
& +\left(1-\frac{\beta_{n}}{1-\alpha_{n}}\right) d\left(x_{n}, q\right) \\
\leq & \frac{\beta_{n}}{1-\alpha_{n}}\left(1+h_{n}\right) d\left(I_{i}\left(P I_{i}\right)^{n-1} z_{n}, q\right)+s_{n} \\
& +\left(1-\frac{\beta_{n}}{1-\alpha_{n}}\right) d\left(x_{n}, q\right) \\
\leq & \frac{\beta_{n}}{1-\alpha_{n}}\left(1+h_{n}\right)^{2} d\left(x_{n}, q\right)+\left(1+h_{n}\right) s_{n}+s_{n} \\
& +\left(1-\frac{\beta_{n}}{1-\alpha_{n}}\right) d\left(x_{n}, q\right) \\
\leq & \left(1+h_{n}\right)^{2} d\left(x_{n}, q\right)+\left(1+h_{n}\right) s_{n}+s_{n} .
\end{aligned}
$$

Therefore

$$
\limsup _{n \rightarrow \infty} d\left(P W\left(T_{i}\left(P T_{i}\right)^{n-1} z_{n}, x_{n}, \frac{\beta_{n}}{1-\alpha_{n}}\right), q\right) \leq c .
$$

As $\lim _{n \rightarrow \infty} d\left(x_{n+1}, q\right)=c$, we have

$$
\lim _{n \rightarrow \infty} d\left(W\left(T_{i}\left(P T_{i}\right)^{n-1} y_{n}, P W\left(T_{i}\left(P T_{i}\right)^{n-1} z_{n}, x_{n}, \frac{\beta_{n}}{1-\alpha_{n}}\right), \alpha_{n}\right), q\right)=c .
$$

It follows from Lemma 2.4 and the sequences (3.5)-(3.7) that

$$
\lim _{n \rightarrow \infty} d\left(T_{i}\left(P T_{i}\right)^{n-1} y_{n}, P W\left(T_{i}\left(P T_{i}\right)^{n-1} z_{n}, x_{n}, \frac{\beta_{n}}{1-\alpha_{n}}\right)\right)=0 .
$$

(ii) Assume $0<\liminf _{n \rightarrow \infty} b_{n} \leq \limsup _{n \rightarrow \infty}\left(b_{n}+c_{n}\right)<1$, then there exist $\tau_{1}, \tau_{2} \in(0,1)$ such that $0<\tau_{1} \leq b_{n} \leq b_{n}+c_{n} \leq \tau_{2}<1$ for all $n \geq 1$. Next we calculate:

$$
\begin{aligned}
d\left(x_{n+1}, q\right)= & d\left(P W\left(T_{i}\left(P T_{i}\right)^{n-1} y_{n}, P W\left(T_{i}\left(P T_{i}\right)^{n-1} z_{n}, x_{n}, \frac{\beta_{n}}{1-\alpha_{n}}\right), \alpha_{n}\right), q\right) \\
\leq & d\left(W\left(T_{i}\left(P T_{i}\right)^{n-1} y_{n}, P W\left(T_{i}\left(P T_{i}\right)^{n-1} z_{n}, x_{n}, \frac{\beta_{n}}{1-\alpha_{n}}\right), \alpha_{n}\right), q\right) \\
\leq & \alpha_{n} d\left(T_{i}\left(P T_{i}\right)^{n-1} y_{n}, q\right) \\
& +\left(1-\alpha_{n}\right) d\left(P W\left(T_{i}\left(P T_{i}\right)^{n-1} z_{n}, x_{n}, \frac{\beta_{n}}{1-\alpha_{n}}\right), q\right) \\
\leq & \alpha_{n} d\left(T_{i}\left(P T_{i}\right)^{n-1} y_{n}, q\right) \\
& +\left(1-\alpha_{n}\right) d\left(T_{i}\left(P T_{i}\right)^{n-1} y_{n}, P W\left(T_{i}\left(P T_{i}\right)^{n-1} z_{n}, x_{n}, \frac{\beta_{n}}{1-\alpha_{n}}\right)\right) \\
& +\left(1-\alpha_{n}\right) d\left(T_{i}\left(P T_{i}\right)^{n-1} y_{n}, q\right)
\end{aligned}
$$




$$
\begin{aligned}
\leq & \left(1+h_{n}\right) d\left(I_{i}\left(P I_{i}\right)^{n-1} y_{n}, q\right)+s_{n} \\
& +d\left(T_{i}\left(P T_{i}\right)^{n-1} y_{n}, P W\left(T_{i}\left(P T_{i}\right)^{n-1} z_{n}, x_{n}, \frac{\beta_{n}}{1-\alpha_{n}}\right)\right) \\
\leq & \left(1+h_{n}\right)^{2} d\left(y_{n}, q\right)+\left(2+h_{n}\right) s_{n} \\
& +d\left(T_{i}\left(P T_{i}\right)^{n-1} y_{n}, P W\left(T_{i}\left(P T_{i}\right)^{n-1} z_{n}, x_{n}, \frac{\beta_{n}}{1-\alpha_{n}}\right)\right) .
\end{aligned}
$$

That is,

$$
\begin{aligned}
d\left(x_{n+1}, q\right) \leq & \left(1+h_{n}\right)^{2} d\left(y_{n}, q\right)+\left(2+h_{n}\right) s_{n} \\
& +d\left(T_{i}\left(P T_{i}\right)^{n-1} y_{n}, P W\left(T_{i}\left(P T_{i}\right)^{n-1} z_{n}, x_{n}, \frac{\beta_{n}}{1-\alpha_{n}}\right)\right) .
\end{aligned}
$$

Applying liminf in the above inequality and then using (3.8), we have

$$
c \leq \liminf _{n \rightarrow \infty} d\left(y_{n}, q\right) \leq \limsup _{n \rightarrow \infty} d\left(y_{n}, q\right) \leq c .
$$

That is,

$$
\lim _{n \rightarrow \infty} d\left(P W\left(T_{i}\left(P T_{i}\right)^{n-1} z_{n}, P W\left(T_{i}\left(P T_{i}\right)^{n-1} x_{n}, x_{n}, \frac{c_{n}}{1-b_{n}}\right), b_{n}\right), q\right)=c .
$$

It follows from (3.3) and (3.9) that

$$
\lim _{n \rightarrow \infty} d\left(W\left(T_{i}\left(P T_{i}\right)^{n-1} z_{n}, P W\left(T_{i}\left(P T_{i}\right)^{n-1} x_{n}, x_{n}, \frac{c_{n}}{1-b_{n}}\right), b_{n}\right), q\right)=c
$$

Similar to (3.5), we have

$$
\limsup _{n \rightarrow \infty} d\left(T_{i}\left(P T_{i}\right)^{n-1} z_{n}, q\right) \leq c
$$

Similar to (3.6), we have

$$
\limsup _{n \rightarrow \infty} d\left(P W\left(T_{i}\left(P T_{i}\right)^{n-1} x_{n}, x_{n}, \frac{c_{n}}{1-b_{n}}\right), q\right) \leq c
$$

The sequences in (3.10), (3.11), and (3.12) satisfy the hypotheses of Lemma 2.4, therefore it follows that

$$
\lim _{n \rightarrow \infty} d\left(T_{i}\left(P T_{i}\right)^{n-1} z_{n}, P W\left(T_{i}\left(P T_{i}\right)^{n-1} x_{n}, x_{n}, \frac{c_{n}}{1-b_{n}}\right)\right)=0 .
$$

(iii) As $0<\liminf _{n \rightarrow \infty} b_{n} \leq \limsup _{n \rightarrow \infty}\left(b_{n}+c_{n}\right)<1$, so as in part (ii), there exist $\tau_{1}, \tau_{2} \in$ $(0,1)$ such that $0<\tau_{1} \leq b_{n} \leq b_{n}+c_{n} \leq \tau_{2}<1$ for all $n \geq 1$. Also $0<\liminf _{n \rightarrow \infty} a_{n} \leq$ $\limsup _{n \rightarrow \infty} a_{n}<1$ shows that there exist $\delta_{1}, \delta_{2} \in(0,1)$ such that $0<\delta_{1} \leq b_{n} \leq b_{n}+c_{n} \leq$ $\delta_{2}<1$ for all $n \geq 1$. 
Taking liminf in the inequality

$$
\begin{aligned}
d\left(y_{n}, q\right)= & d\left(P W\left(T_{i}\left(P T_{i}\right)^{n-1} z_{n}, P W\left(T_{i}\left(P T_{i}\right)^{n-1} x_{n}, x_{n}, \frac{c_{n}}{1-b_{n}}\right), b_{n}\right), q\right) \\
\leq & d\left(W\left(T_{i}\left(P T_{i}\right)^{n-1} z_{n}, P W\left(T_{i}\left(P T_{i}\right)^{n-1} x_{n}, x_{n}, \frac{c_{n}}{1-b_{n}}\right), b_{n}\right), q\right) \\
\leq & b_{n} d\left(T_{i}\left(P T_{i}\right)^{n-1} z_{n}, q\right)+\left(1-b_{n}\right) d\left(P W\left(T_{i}\left(P T_{i}\right)^{n-1} x_{n}, x_{n}, \frac{c_{n}}{1-b_{n}}\right), q\right) \\
\leq & b_{n} d\left(T_{i}\left(P T_{i}\right)^{n-1} z_{n}, q\right)+\left(1-b_{n}\right) d\left(T_{i}\left(P T_{i}\right)^{n-1} z_{n}, q\right) \\
& +\left(1-b_{n}\right) d\left(T_{i}\left(P T_{i}\right)^{n-1} z_{n}, P W\left(T_{i}\left(P T_{i}\right)^{n-1} x_{n}, x_{n}, \frac{c_{n}}{1-b_{n}}\right)\right) \\
\leq & \left(1+h_{n}\right)^{2} d\left(z_{n}, q\right)+\left(2+h_{n}\right) s_{n} \\
& +d\left(T_{i}\left(P T_{i}\right)^{n-1} z_{n}, P W\left(T_{i}\left(P T_{i}\right)^{n-1} x_{n}, x_{n}, \frac{c_{n}}{1-b_{n}}\right)\right)
\end{aligned}
$$

and using (3.13), we have

$$
c \leq \liminf _{n \rightarrow \infty} d\left(z_{n}, q\right) \leq \limsup _{n \rightarrow \infty} d\left(z_{n}, q\right) \leq c .
$$

Since

$$
d\left(z_{n}, q\right)=d\left(P W\left(I_{i}\left(P I_{i}\right)^{n-1} x_{n}, x_{n}, a_{n}\right), q\right) \leq d\left(W\left(I_{i}\left(P I_{i}\right)^{n-1} x_{n}, x_{n}, a_{n}\right), q\right),
$$

we have

$$
\lim _{n \rightarrow \infty} d\left(W\left(I_{i}\left(P I_{i}\right)^{n-1} x_{n}, x_{n}, a_{n}\right), q\right)=c
$$

Appealing to Lemma 2.4, we have

$$
\lim _{n \rightarrow \infty} d\left(I_{i}\left(P I_{i}\right)^{n-1} x_{n}, x_{n}\right)=0 .
$$

Therefore

$$
\begin{aligned}
d\left(z_{n}, x_{n}\right) & =d\left(P W\left(I_{i}\left(P I_{i}\right)^{n-1} x_{n}, x_{n}, a_{n}\right), x_{n}\right) \\
& \leq d\left(W\left(I_{i}\left(P I_{i}\right)^{n-1} x_{n}, x_{n}, a_{n}\right), x_{n}\right) \\
& \leq a_{n} d\left(I_{i}\left(P I_{i}\right)^{n-1} x_{n}, x_{n}\right) \\
& \leq d\left(I_{i}\left(P I_{i}\right)^{n-1} x_{n}, x_{n}\right) \rightarrow 0
\end{aligned}
$$

Since

$$
\begin{aligned}
& d\left(T_{i}\left(P T_{i}\right)^{n-1} x_{n}, x_{n}\right) \leq d\left(T_{i}\left(P T_{i}\right)^{n-1} x_{n}, T_{i}\left(P T_{i}\right)^{n-1} z_{n}\right)+d\left(T_{i}\left(P T_{i}\right)^{n-1} z_{n}, x_{n}\right) \\
& d\left(T_{i}\left(P T_{i}\right)^{n-1} z_{n}, x_{n}\right) \leq d\left(T_{i}\left(P T_{i}\right)^{n-1} z_{n}, P W\left(T_{i}\left(P T_{i}\right)^{n-1} x_{n}, x_{n}, \frac{c_{n}}{1-b_{n}}\right)\right)
\end{aligned}
$$




$$
\begin{aligned}
& +d\left(P W\left(T_{i}\left(P T_{i}\right)^{n-1} x_{n}, x_{n}, \frac{c_{n}}{1-b_{n}}\right), x_{n}\right) \\
\leq & d\left(T_{i}\left(P T_{i}\right)^{n-1} z_{n}, P W\left(T_{i}\left(P T_{i}\right)^{n-1} x_{n}, x_{n}, \frac{c_{n}}{1-b_{n}}\right)\right) \\
& +\frac{c_{n}}{1-b_{n}} d\left(T_{i}\left(P T_{i}\right)^{n-1} x_{n}, x_{n}\right) .
\end{aligned}
$$

It follows from (3.16) and (3.17) that

$$
\begin{aligned}
(1- & \left.\frac{c_{n}}{1-b_{n}}\right) d\left(T_{i}\left(P T_{i}\right)^{n-1} x_{n}, x_{n}\right) \\
\leq & d\left(T_{i}\left(P T_{i}\right)^{n-1} x_{n}, T_{i}\left(P T_{i}\right)^{n-1} z_{n}\right) \\
& \quad+d\left(T_{i}\left(P T_{i}\right)^{n-1} z_{n}, P W\left(T_{i}\left(P T_{i}\right)^{n-1} x_{n}, x_{n}, \frac{c_{n}}{1-b_{n}}\right)\right) .
\end{aligned}
$$

Therefore, we have

$$
\begin{aligned}
(1- & \left.\frac{\tau_{2}}{1-\tau_{2}}\right) d\left(T_{i}\left(P T_{i}\right)^{n-1} x_{n}, x_{n}\right) \\
\leq & d\left(T_{i}\left(P T_{i}\right)^{n-1} x_{n}, T_{i}\left(P T_{i}\right)^{n-1} z_{n}\right) \\
& \quad+d\left(T_{i}\left(P T_{i}\right)^{n-1} z_{n}, P W\left(T_{i}\left(P T_{i}\right)^{n-1} x_{n}, x_{n}, \frac{c_{n}}{1-b_{n}}\right)\right) \\
\leq & \left(1+h_{n}\right)^{2} d\left(x_{n}, z_{n}\right)+\left(2+h_{n}\right) s_{n} \\
& +d\left(T_{i}\left(P T_{i}\right)^{n-1} z_{n}, P W\left(T_{i}\left(P T_{i}\right)^{n-1} x_{n}, x_{n}, \frac{c_{n}}{1-b_{n}}\right)\right) .
\end{aligned}
$$

It follows from (3.8), (3.15), and (3.19) that

$$
\lim _{n \rightarrow \infty} d\left(T_{i}\left(P T_{i}\right)^{n-1} x_{n}, x_{n}\right)=0 .
$$

This completes the proof.

Theorem 3.3 Under the assumptions of Lemma 3.1, let $T_{i}, I_{i}: K \rightarrow X\left(i \in I_{0}\right)$ be uniformly L-Lipschitzian, the sequence $\left\{x_{n}\right\}$ is generated by (3.1) satisfying the conditions:

(i) $0<\liminf _{n \rightarrow \infty} \alpha_{n} \leq \limsup _{n \rightarrow \infty}\left(\alpha_{n}+\beta_{n}\right)<1$,

(ii) $0<\liminf _{n \rightarrow \infty} b_{n} \leq \limsup _{n \rightarrow \infty}\left(b_{n}+c_{n}\right)<1$,

(iii) $0<\liminf _{n \rightarrow \infty} a_{n} \leq \limsup _{n \rightarrow \infty} a_{n}<1$.

Then $\left\{x_{n}\right\}$ in (3.1) has approximate common fixed point property for $I_{i}, T_{i}\left(i \in I_{0}\right)$.

Proof Since $T_{i}$ is uniformly L-Lipschitzian, it follows from (3.15) and (3.20) that

$$
\begin{aligned}
d\left(T_{i}\left(P T_{i}\right)^{n-1} z_{n}, x_{n}\right) \leq & d\left(T_{i}\left(P T_{i}\right)^{n-1} z_{n}, T_{i}\left(P T_{i}\right)^{n-1} x_{n}\right) \\
& +d\left(T_{i}\left(P T_{i}\right)^{n-1} x_{n}, x_{n}\right) \\
\leq & L d\left(z_{n}, x_{n}\right)+d\left(T_{i}\left(P T_{i}\right)^{n-1} x_{n}, x_{n}\right) \rightarrow 0 .
\end{aligned}
$$


It follows from (3.13) and (3.21) that

$$
\begin{aligned}
d\left(y_{n}, x_{n}\right)= & d\left(P W\left(T_{i}\left(P T_{i}\right)^{n-1} z_{n}, P W\left(T_{i}\left(P T_{i}\right)^{n-1} x_{n}, x_{n}, \frac{c_{n}}{1-b_{n}}\right), b_{n}\right), x_{n}\right) \\
\leq & d\left(W\left(T_{i}\left(P T_{i}\right)^{n-1} z_{n}, P W\left(T_{i}\left(P T_{i}\right)^{n-1} x_{n}, x_{n}, \frac{c_{n}}{1-b_{n}}\right), b_{n}\right), x_{n}\right) \\
\leq & b_{n} d\left(T_{i}\left(P T_{i}\right)^{n-1} z_{n}, x_{n}\right) \\
& +\left(1-b_{n}\right) d\left(P W\left(T_{i}\left(P T_{i}\right)^{n-1} x_{n}, x_{n}, \frac{c_{n}}{1-b_{n}}\right), x_{n}\right) \\
\leq & d\left(T_{i}\left(P T_{i}\right)^{n-1} z_{n}, x_{n}\right) \\
& +d\left(T_{i}\left(P T_{i}\right)^{n-1} z_{n}, P W\left(T_{i}\left(P T_{i}\right)^{n-1} x_{n}, x_{n}, \frac{c_{n}}{1-b_{n}}\right)\right) \\
\rightarrow & 0 .
\end{aligned}
$$

Since $T_{i}$ is uniformly L-Lipschitzian, it follows from (3.13) and (3.22) that

$$
\begin{aligned}
d\left(T_{i}\left(P T_{i}\right)^{n-1} y_{n}, x_{n}\right) & \leq d\left(T_{i}\left(P T_{i}\right)^{n-1} y_{n}, T_{i}\left(P T_{i}\right)^{n-1} x_{n}\right)+d\left(T_{i}\left(P T_{i}\right)^{n-1} x_{n}, x_{n}\right) \\
& \leq L d\left(y_{n}, x_{n}\right)+d\left(T_{i}\left(P T_{i}\right)^{n-1} x_{n}, x_{n}\right) \rightarrow 0
\end{aligned}
$$

It follows from (3.8) and (3.23) that

$$
\begin{aligned}
d\left(x_{n+1}, x_{n}\right)= & d\left(P W\left(T_{i}\left(P T_{i}\right)^{n-1} y_{n}, P W\left(T_{i}\left(P T_{i}\right)^{n-1} z_{n}, x_{n}, \frac{\beta_{n}}{1-\alpha_{n}}\right), \alpha_{n}\right), x_{n}\right) \\
\leq & d\left(W\left(T_{i}\left(P T_{i}\right)^{n-1} y_{n}, P W\left(T_{i}\left(P T_{i}\right)^{n-1} z_{n}, x_{n}, \frac{\beta_{n}}{1-\alpha_{n}}\right), \alpha_{n}\right), x_{n}\right) \\
\leq & \alpha_{n} d\left(T_{i}\left(P T_{i}\right)^{n-1} y_{n}, x_{n}\right) \\
& +\left(1-\alpha_{n}\right) d\left(P W\left(T_{i}\left(P T_{i}\right)^{n-1} z_{n}, x_{n}, \frac{\beta_{n}}{1-\alpha_{n}}\right), x_{n}\right) \\
\leq & d\left(T_{i}\left(P T_{i}\right)^{n-1} y_{n}, x_{n}\right) \\
& +d\left(T_{i}\left(P T_{i}\right)^{n-1} y_{n}, P W\left(T_{i}\left(P T_{i}\right)^{n-1} z_{n}, x_{n}, \frac{\beta_{n}}{1-\alpha_{n}}\right)\right) \\
\rightarrow & 0 \quad \text { as } n \rightarrow \infty .
\end{aligned}
$$

Note that the inequality

$$
\begin{aligned}
d\left(x_{n}, T_{i} x_{n}\right) \leq & d\left(x_{n}, x_{n+1}\right)+d\left(x_{n+1}, T_{i}\left(P T_{i}\right)^{n} x_{n+1}\right) \\
& +d\left(T_{i}\left(P T_{i}\right)^{n} x_{n+1}, T_{i}\left(P T_{i}\right)^{n} x_{n}\right)+d\left(T_{i}\left(P T_{i}\right)^{n} x_{n}, T_{i} x_{n}\right) \\
= & d\left(x_{n}, x_{n+1}\right)+d\left(T_{i}\left(P T_{i}\right)^{n} x_{n+1}, T_{i}\left(P T_{i}\right)^{n} x_{n}\right) \\
& +d\left(x_{n+1}, T_{i}\left(P T_{i}\right)^{n} x_{n+1}\right)+d\left(T_{i}\left(P T_{i}\right)^{n} x_{n}, T_{i} x_{n}\right) \\
\leq & (1+L) d\left(x_{n}, x_{n+1}\right)+d\left(x_{n+1}, T_{i}\left(P T_{i}\right)^{n} x_{n+1}\right)+L d\left(T_{i}\left(P T_{i}\right)^{n-1} x_{n}, x_{n}\right)
\end{aligned}
$$


together with (3.20) and (3.24) gives

$$
\lim _{n \rightarrow \infty} d\left(x_{n}, T_{i} x_{n}\right)=0 \quad \text { for all } i \in I_{0} .
$$

Similarly, we can prove that

$$
\lim _{n \rightarrow \infty} d\left(x_{n}, I_{i} x_{n}\right)=0 \quad \text { for all } i \in I_{0}
$$

Equations (3.25) and (3.26) prove that $\left\{x_{n}\right\}$ has approximate common fixed point property for $I_{i}, T_{i}\left(i \in I_{0}\right)$. This completes the proof.

Theorem 3.4 Under the assumptions of Theorem 3.3, if $\left\{T_{i}: i \in I_{0}\right\}$ and $\left\{I_{i}: i \in I_{0}\right\}$ satisfy condition $(B)$ with respect to the sequence $\left\{x_{n}\right\}$, then $\left\{x_{n}\right\}$ in (3.1) converges strongly to a common fixed point of $\left\{T_{i}: i \in I_{0}\right\}$ and $\left\{I_{i}: i \in I_{0}\right\}$.

Proof It follows from Theorem 3.3 that

$$
\lim _{n \rightarrow \infty} d\left(x_{n}, T_{i} x_{n}\right)=\lim _{n \rightarrow \infty} d\left(x_{n}, I_{i} x_{n}\right)=0, \quad \forall i \in I_{0} .
$$

Since $\left\{T_{i}: i \in I_{0}\right\}$ and $\left\{I_{i}: i \in I_{0}\right\}$ satisfy condition (B) with respect to the sequence $\left\{x_{n}\right\}$, we have

$$
\lim _{n \rightarrow \infty} d\left(x_{n}, \mathcal{F}\right)=0 .
$$

Next, we show that $\left\{x_{n}\right\}$ is a Cauchy sequence. For any $q \in \mathcal{F}$. It follows from (3.4) that there exists a positive constant $M$ such that

$$
d\left(x_{n+1}, q\right) \leq d\left(x_{n}, q\right)+\gamma_{n}
$$

where $\gamma_{n}=M \rho_{n}$, and $\sum_{n=1}^{\infty} \gamma_{n}<\infty$. For an arbitrary $\epsilon>0$, since $\lim _{n \rightarrow \infty} d\left(x_{n}, \mathcal{F}\right)=0$ and $\sum_{n=1}^{\infty} \gamma_{n}<\infty$, there exists a positive integer $N$ such that $d\left(x_{n}, \mathcal{F}\right) \leq \epsilon / 4, \sum_{j=n}^{\infty} \gamma_{j} \leq \epsilon / 4$ for all $n \geq N$. So, we have $d\left(x_{N}, \mathcal{F}\right) \leq \epsilon / 4, \sum_{j=N}^{\infty} \gamma_{j} \leq \epsilon / 4$. This means that there exists a $q_{1} \in \mathcal{F}$ such that $d\left(x_{N}, q_{1}\right) \leq \epsilon / 4$. It follows from (3.27) that when $n \geq N$,

$$
\begin{aligned}
d\left(x_{n+m}, x_{n}\right) & \leq d\left(x_{n+m}, q_{1}\right)+d\left(x_{n}, q_{1}\right) \\
& \leq d\left(x_{N}, q_{1}\right)+\sum_{j=N}^{n+m-1} \gamma_{j}+d\left(x_{N}, q_{1}\right)+\sum_{j=N}^{n-1} \gamma_{j} \\
& \leq 2\left(d\left(x_{N}, q_{1}\right)+\sum_{j=N}^{\infty} \gamma_{j}\right) \\
& \leq 2\left(\frac{\epsilon}{4}+\frac{\epsilon}{4}\right)=\epsilon .
\end{aligned}
$$

This implies that $\left\{x_{n}\right\}$ is a Cauchy sequence. $K$ is complete for it is a closed subset in a complete hyperbolic space. Without loss of generality, we can assume that $\left\{x_{n}\right\}$ converges strongly to some point $q^{*} \in K$. It is easy to prove that $\mathcal{F}$ is closed. It follows from $\lim _{n \rightarrow \infty} d\left(x_{n}, \mathcal{F}\right)=0$ that $q^{*} \in \mathcal{F}$. This completes the proof. 
Theorem 3.5 Under the assumptions of Theorem 3.3, then the sequence $\left\{x_{n}\right\}$ defined by (3.1) $\triangle$-converges to a point in $\mathcal{F}$.

Proof It follows from Lemma 3.1 that $\left\{x_{n}\right\}$ is bounded. Therefore by Lemma 2.3, $\left\{x_{n}\right\}$ has a unique asymptotic center, that is, $A\left(\left\{x_{n}\right\}\right)=\{x\}$. Let $\left\{u_{n}\right\}$ be any subsequence of $\left\{x_{n}\right\}$ such that $A\left(\left\{u_{n}\right\}\right)=\{u\}$. By (3.25) and (3.26), we have $\lim _{n \rightarrow \infty} d\left(u_{n}, T_{i} u_{n}\right)=\lim _{n \rightarrow \infty} d\left(u_{n}, I_{i} u_{n}\right)=$ 0 for $i \in I_{0}$. We claim that $u$ is the common fixed point of $\left\{T_{i}: i \in I_{0}\right\}$ and $\left\{I_{i}: i \in I_{0}\right\}$.

To do this, we define a sequence $\left\{z_{n}\right\}$ in $K$ by $z_{n}=T_{i}\left(P T_{i}\right)^{n-1} u$. Observe that

$$
\begin{aligned}
d\left(z_{n}, u_{n}\right) & \leq d\left(T_{i}\left(P T_{i}\right)^{n-1} u, T_{i}\left(P T_{i}\right)^{n-1} u_{n}\right)+d\left(T_{i}\left(P T_{i}\right)^{n-1} u_{n}, u_{n}\right) \\
& \leq\left(1+h_{n}\right) d\left(I_{i}\left(P I_{i}\right)^{n-1} u, I_{i}\left(P I_{i}\right)^{n-1} u_{n}\right)+s_{n}+d\left(T_{i}\left(P T_{i}\right)^{n-1} u_{n}, u_{n}\right) \\
& \leq\left(1+h_{n}\right)^{2} d\left(u, u_{n}\right)+\left(2+h_{n}\right) s_{n}+d\left(T_{i}\left(P T_{i}\right)^{n-1} u_{n}, u_{n}\right) .
\end{aligned}
$$

Therefore, we have

$$
\widetilde{r}\left(z_{n},\left\{u_{n}\right\}\right)=\limsup _{n \rightarrow \infty} d\left(z_{n}, u_{n}\right) \leq \limsup _{n \rightarrow \infty} d\left(u, u_{n}\right)=\widetilde{r}\left(u,\left\{u_{n}\right\}\right) .
$$

This implies that $\left|\widetilde{r}\left(z_{n},\left\{u_{n}\right\}\right)-\widetilde{r}\left(u,\left\{u_{n}\right\}\right)\right| \rightarrow o$ as $n \rightarrow \infty$. It follows from Lemma 2.5 that $\lim _{n \rightarrow \infty} T_{i}\left(P T_{i}\right)^{n-1} u=u$. Since $K$ is closed, $\lim _{n \rightarrow \infty} T_{i}\left(P T_{i}\right)^{n-1} u=u \in K$ and $\lim _{n \rightarrow \infty} T_{i}\left(P T_{i}\right)^{n} u=T_{i} u$, that is, $T_{i} u=u$. Similarly, we can show that $u$ is the common fixed point of $\left\{I_{i}: i \in I_{0}\right\}$. Therefore $u$ is the common fixed point of $\left\{T_{i}: i \in I_{0}\right\}$ and $\left\{I_{i}: i \in I_{0}\right\}$. It follows from Lemma 3.1 that $\lim _{n \rightarrow \infty} d\left(x_{n}, u\right)$ exists. Suppose $x \neq u$. Then by the uniqueness of asymptotic centers, we have

$$
\begin{array}{r}
\limsup _{n \rightarrow \infty} d\left(u_{n}, u\right)<\limsup _{n \rightarrow \infty} d\left(u_{n}, x\right) \leq \limsup _{n \rightarrow \infty} d\left(x_{n}, x\right) \\
<\limsup _{n \rightarrow \infty} d\left(x_{n}, u\right)=\limsup _{n \rightarrow \infty} d\left(u_{n}, u\right),
\end{array}
$$

which gives a contradiction. Hence $x=u$.

Therefore $A\left(\left\{u_{n}\right\}\right)=\{u\}$ for all subsequences $\left\{u_{n}\right\}$ of $\left\{x_{n}\right\}$. This proves that $\left\{x_{n}\right\} \Delta$ converges to a point in $\mathcal{F}$.

Example 3.6 Let us consider that $\mathbb{R}$, the set of real number with the usual norm $|\cdot|$. Let $K=\left[-\frac{2}{3}, \frac{2}{3}\right] \subset \mathbb{R}$. The mapping $T_{1}, T_{2}: K \rightarrow K$ are defined by

$$
T_{1} x= \begin{cases}\sin \frac{x}{3}, & x \in\left[-\frac{2}{3}, 0\right], \\ -\sin \frac{x}{3}, & x \in\left(0, \frac{2}{3}\right],\end{cases}
$$

and

$$
T_{2} x= \begin{cases}x, & x \in\left[-\frac{2}{3}, 0\right], \\ x^{4}, & x \in\left(0, \frac{2}{3}\right] .\end{cases}
$$


Then $T_{1}$ and $T_{2}$ are generalized asymptotically nonexpansive mappings. Note that $F\left(T_{1}\right)=$ $\{0\}$ and $F\left(T_{2}\right)=\left\{-\frac{2}{3} \leq x \leq 0\right\}$ and $\mathcal{F}=F\left(T_{1}\right) \cap F\left(T_{2}\right)=\{0\}$. Let

$$
a_{n}=\frac{n}{n+1}, \quad b_{n}=\frac{n}{2 n+1}, \quad c_{n}=\frac{n}{3 n+1}, \quad \alpha_{n}=\frac{n}{4 n+1}, \quad \beta_{n}=\frac{n}{5 n+1}
$$

for all $n \geq 1$. Therefore, the conditions of Theorem 3.5 are fulfilled.

Theorem 3.7 Under the assumptions of Theorem 3.3, $\left\{x_{n}\right\}$ in (3.1) converges strongly to a common fixed point of $\left\{T_{i}: i \in I_{0}\right\}$ and $\left\{I_{i}: i \in I_{0}\right\}$ if and only if $\liminf _{n \rightarrow \infty} d\left(x_{n}, \mathcal{F}\right)=0$.

Proof The necessity is obvious. Indeed, if $x_{n} \rightarrow q \in \mathcal{F}$ as $n \rightarrow \infty$, then

$$
d\left(x_{n}, \mathcal{F}\right)=\inf _{q \in \mathcal{F}} d\left(x_{n}, q\right) \leq d\left(x_{n}, q\right) \rightarrow 0 \quad(\text { as } n \rightarrow \infty) .
$$

Now, we show sufficiency. Equation (3.27) means that

$$
\inf _{q \in \mathcal{F}} d\left(x_{n+1}, q\right) \leq \inf _{q \in \mathcal{F}} d\left(x_{n}, q\right)+\gamma_{n}
$$

that is,

$$
d\left(x_{n+1}, \mathcal{F}\right) \leq d\left(x_{n}, \mathcal{F}\right)+\gamma_{n} .
$$

It follows from (3.28), Lemma 2.6, and $\liminf _{n \rightarrow \infty} d\left(x_{n}, \mathcal{F}\right)=0$ that $\lim _{n \rightarrow \infty} d\left(x_{n}, \mathcal{F}\right)=0$. Thus, the rest of the proof follows as in the proof of Theorem 3.4. This completes the proof.

Theorem 3.8 Under the assumptions of Theorem 3.3, if at least one mapping of the mappings $\left\{T_{i}: i \in I_{0}\right\}$ and $\left\{I_{i}: i \in I_{0}\right\}$ is semi-compact, then $\left\{x_{n}\right\}$ in (3.1) converges strongly to a common fixed point of $\left\{T_{i}: i \in I_{0}\right\}$ and $\left\{I_{i}: i \in I_{0}\right\}$.

Proof Without loss of generality, we may assume that $T_{1}$ is semi-compact. By (3.25) and the assumption that $T_{1}$ is semi-compact, there exists a subsequence $\left\{x_{n_{j}}\right\} \subset\left\{x_{n}\right\}$ such that $\left\{x_{n_{j}}\right\}$ converges strongly to some point $q \in K$. Then by the continuity of $T_{i}\left(i \in I_{0}\right)$, we get

$$
d\left(q, T_{i} q\right)=\lim _{j \rightarrow \infty} d\left(x_{n_{j}}, T_{i} x_{n_{j}}\right)=0, \quad i \in I_{0} .
$$

Similarly, we can prove $d\left(q, I_{i} q\right)=0$ for $i \in I_{0}$. Therefore $q \in \mathcal{F}$. It follows from Lemma 3.1 that $\lim _{n \rightarrow \infty} d\left(x_{n}, q\right)$ exists and thus $\lim _{n \rightarrow \infty} d\left(x_{n}, q\right)=0$. This completes the proof.

\section{Competing interests}

The author declares that he has no competing interests.

Author's contributions

The author only contributed to the writing of this paper. The author read and approved the final manuscript.

\section{Acknowledgements}

This work was supported by the Humanity and Social Science Planning Foundation of Ministry of Education of China (Grand No. 14YJAZH095), the National Natural Science Foundation of China (Grand No. 61374081), the Natural Science Foundation of Guangdong Province (Grand No. 2015A030313485, S2013010013034) and the High-level Talents Project in Guangdong Province (Grant No. 2014011). 


\section{References}

1. Temir, S: On the convergence theorems of implicit iteration process for a finite family of $/$-asymptotically nonexpansive mappings. J. Comput. Appl. Math. 225, 398-405 (2009)

2. Temir, S, Gul, O: Convergence theorem for I-asymptotically quasi-nonexpansive mapping in Hilbert space. J. Math. Anal. Appl. 329, 759-765 (2007)

3. Khan, $\mathrm{SH}$, Fukhar-ud-din, $\mathrm{H}$ : Weak and strong convergence of a scheme with errors for two nonexpansive mappings. Nonlinear Anal. 61, 1295-1301 (2005)

4. Shahzad, N: Approximating fixed points of non-self nonexpansive mappings in Banach spaces. Nonlinear Anal. 61, 1031-1039 (2005)

5. Chidume, CE, Ofoedu, EU, Zegeye, H: Strong and weak convergence theorems for asymptotically nonexpansive mappings. J. Math. Anal. Appl. 280, 364-374 (2003)

6. Chidume, CE, Shahzad, N, Zegeye, $\mathrm{H}$ : Convergence theorems for mappings which are asymptotically nonexpansive in the intermediate sense. Numer. Funct. Anal. Optim. 25, 239-257 (2004)

7. Wang, L: Strong and weak convergence theorems for common fixed points of nonself asymptotically nonexpansive mappings. J. Math. Anal. Appl. 323, 550-557 (2006)

8. Yang, L: Modified multistep iterative process for some common fixed point of a finite family of nonself asymptotically nonexpansive mappings. Math. Compt. Modelling 45, 1157-1169 (2007)

9. Thianwan, S: Common fixed points of new iterations for two asymptotically nonexpansive nonself mappings in a Banach space. J. Comput. Appl. Math. 224, 688-695 (2009)

10. Kohlenbach, U: Some logical metatheorems with applications in functional analysis. Trans. Am. Math. Soc. 357, 89-128 (2005)

11. Takahashi, W: A convexity in metric spaces and nonexpansive mappings. Kodai Math. Semin. Rep. 22, 142-149 (1970)

12. Shimizu, T, Takahashi, W: Fixed points of multivalued mappings in certain convex metric spaces. Topol. Methods Nonlinear Anal. 8, 197-203 (1996)

13. Lim, TC: Remarks on some fixed point theorems. Proc. Am. Math. Soc. 60, 179-182 (1976)

14. Dhompongsa, S, Panyanak, B: On $\triangle$-convergence theorems in CAT(0)-spaces. Comput. Math. Appl. 56(10), 2572-2579 (2008)

15. Khan, AR, Fukhar-ud-din, H, Khan, MAA: An implicit algorithm for two finite families of nonexpansive maps in hyperbolic spaces. Fixed Point Theory Appl. 2012, 54 (2012)

16. Tan, KK, Xu, HK: Approximating fixed points of nonexpansive by the Ishikawa iteration process. J. Math. Anal. Appl. $178,301-308$ (1993)

\section{Submit your manuscript to a SpringerOpen ${ }^{\odot}$ journal and benefit from:}

- Convenient online submission

- Rigorous peer review

- Immediate publication on acceptance

- Open access: articles freely available online

- High visibility within the field

- Retaining the copyright to your article 